\title{
Analysis of Time-dependent Reliability of Concrete Gravity Dam Damage Based on Gray Theory
}

\author{
Fanghua Liu and Hezhi Li
}

Nanchang Key Laboratory of Material and Structure Detection, Jiangxi University of Technology

Keywords: Time-dependent Reliability; Gray Theory; Concrete Damage; Concrete Gravity Dam

\begin{abstract}
This paper applies the reliability theory, carries out time-dependent reliability over concrete gravity dam based on gray theory and damage theory, and applies the theory to practical engineering. Specific work are: 1. Apply the gray theory, build the GM model to analyze, supplement and predict the change and development laws of gravity dam random variables, and apply to structural reliability analysis for analysis of time-dependent reliability over the structure. 2 . Adopt the damage mechanics theory, introduce the damage variable, build a new function based on four-parameter concrete model, reflect the concrete structure and the damage change features during the use, and thus analyze the structural time-dependent reliability at each period of time. 3. Carry out Taylor expansion to quadratic nonlinear term over the structural functional equation, combine with the engineering application principle, and obtain a feasible reliability utility computing method. This method does not need iteration, so it has greatly reduced the amount of computation, and has higher accuracy than the traditional first-order second-moment method.
\end{abstract}

\section{Introduction}

Structural reliability is a safety issue of studied structure in various factors, including the structural safety, serviceability, durability, maintainability, storability and combinations, that is, the ability of completing the required function under prescribed conditions and within the specified time. Reliability means the probability of completing the specified function under prescribed conditions and within the specified time.

When applying the reliability calculation method through mathematical model, it is assumed that the reliability changes follow the experimentally determined statistic law. Firstly, regard the reliability as the amount of time range, and reliability changes over time according to certain laws. For example, when studying the fatigue life of structure, the result can be well matched with experimental facts. Secondly, deem the reliability as the result of certain causal factors, and reliability can be seen as a probability of random event for calculation. Use once at a time, which cannot be applied to time features. Its defect lies in no possibility of eliminating failure because the failure reasons are not clarified. Physical reason law is to establish the stress-strength model, considering that the structural stress and strength imposed on structure are random variables (or random process, and sometimes random field), and it obey a certain distribution.

During the long-term structural use, owing to little parameter characteristic test statistics at different times, there is also rare time-dependent analysis and researches. Currently, the reliability researches at the normal period for use are expanded to reliability researches at whole process of life structure, which further provides references to the durability of structure design, or provides the basis 
for maintenance and reinforcement of existing projects. How to assess the safety during the current damaged structure period and predict the reliability of remaining structural service life in the case of damage and how to take preventive measures are urgent engineering problems to be solved. Therefore, reasonable research and analysis of structural time-dependent reliability have a very important guiding significance for the engineering structure during the construction and use period as well as the whole life process.

In this paper, the main development directions are:

(1) Application of modified materials and new materials in structural engineering.

(2) Note the new structure and special features.

(3) Prevention of structural disaster prevention and structural failure.

(4) Stress the environmental effects of disasters as well as structural performance and reaction control under extreme conditions, prevent abnormal structural failure, and prolong necessary structural life.

(5) Theory and methods of structural engineering design and quality control.

(6) Future engineering system design feature is the scientific information processing, large-system multi-objective optimization, self-control structural behavior and artificial intelligent design.

(7) Close combination between the structure theory and engineering practice.

(8) The structural engineering material develops tow ards "light weight, high strength and multiple functions".

\section{Application of Gray Theory in Structural Reliability Analysis}

Since it is hard and impossible to collect complete concrete material aging test or measured data, this paper uses gray theory to build the concrete aging GM prediction model, so as to predict the aging rules of concrete materials and provide the necessary data for the reliability analysis.

Under the normal distribution condition, the reliability index of random variables is expressed as:

$$
\begin{gathered}
\tilde{\beta}(\mathrm{t})=\frac{\mathrm{u}_{\overline{\mathrm{z}}}}{\sigma_{\overline{\mathrm{z}}}} \\
\tilde{\mathrm{Z}}=\mathrm{R}(\mathrm{t})-\mathrm{S}(\mathrm{t})=\mathrm{g}\left(\tilde{\mathrm{x}}_{1}(\mathrm{t}), \quad \tilde{\mathrm{x}}_{2}(\mathrm{t}) \cdots \cdots \tilde{\mathrm{x}}_{\mathrm{n}}(\mathrm{t})\right)
\end{gathered}
$$

Introduce gray theory in the time-dependent reliability, which is mainly used for the following three aspects:

(1) Regard the structure as a gray system, build $\mathrm{MG}(1, \mathrm{n})$ model according to the known relationship between input and output, and solve the corresponding output by a output system.

(2) Build MG(l,n) model through the statistical law of a certain variable, predict its future trends, estimate the future changes in parameters or system, and conduct gray calculation error estimates through the known value of a variable.

(4) Predict the abnormal event for the structural reliability development behavior characteristic value exceeding (or not reaching) the described value over the existing information, build GM model and conduct disaster prediction. 


\section{Neural Network and Improved Response Surface Method based on Matlab Platform}

Currently, in the practical application of artificial neural networks, most of the neural networks adopt BP networks and its variations. Here, BP network is mainly used for function approximation, which trains a network to approach a function with the input vector and the corresponding output vectors. BP network has higher-order nonlinear analog function, it is possible to fit the input and output according to Matlba neural network design, and complete its mapping function.

When the structure is very complicated, response surface method can solve that it is difficult for the function to have an explicit expression, this method can be decomposed into two main processes: The first is to look for the design point, and the second is to use the known data for function composition within the limited field of design point. On Matlab platform, build the reliability index optimization model, use artificial neural network for training and fitting, obtain the improved response surface method on this basis, and thus solve the difficulty in explicit expression of functions. It is proposed to analyze from the following two aspects:

(1) Build an optimization model to solve reliability index, and select the checking point to fit the design point. In the structural reliability analysis, the geometric meaning of reliability index is the shortest distance between the origins to limit state surface in standard normal coordinates. The pedal point is the checking point. Accordingly, apply Matlba to solve the constraint nonlinear programming,

Build the below optimization model for reliability index:

In the surface:

Make the shortest distance from the origin to this point

Where, is the basic variable, vlb and vub are upper and lower limit of $\mathrm{xi}, \mathrm{D}$ is the failure region, and $\mathrm{g}(\mathrm{x})$ is the performance function.

According to the relationship between input and output in the vicinity of the design point, the artificial neural networks can be used to complete its one-to-one mapping function. Since the artificial neural network has a strong high-order nonlinear fitting function, it can overcome the limitations of quadratic multiple-term hypersurface.

Improved response surface method is as the following:

(1) Apply stochastic finite element method for the analysis of finite-order finite element, and obtain a set of input and output data of structural system.

(2) Establish the artificial neural network to fit the performance function according to the known system input and output data.

(3) Establish concrete damage model, and solve the design checking point through optimization model.

(4) Build the reliability performance function in the vicinity of design checking point of artificial neural network.

(5) Build the optimization model for reliability calculations by using the performance function.

\section{Concrete structure reliability analysis method}

In the structural construction and use process, it will inevitably produce internal or minor structural or surface defects (such as cracks). Under certain external factors (such as load, temperature and corrosion), these defects will continue to expand and merge, form macroscopic cracks, then continue to expand, and eventually lead to structural rupture and damage.

Density change measurement method: 


$$
\mathrm{D}=\frac{\pi \mathrm{r}^{2}}{\pi\left(\mathrm{R}^{3}+\mathrm{r}^{3}\right)^{2 / 3}}=\left(\frac{r^{3}}{R^{3}+r^{3}}\right)^{2 / 3}=\left(1-\frac{\tilde{\rho}}{\rho}\right)^{2 / 3}
$$

Elastic Modulus descent method:

$$
\mathrm{D}=1-\frac{\tilde{\mathrm{E}}}{\mathrm{E}}
$$

Non-uniform microstructure of concrete, there are micro-cracks, sometimes macroscopic defects and cracks pattern, the existence and its concrete defect sensitivity can lead to fracture, depending on the nature of the material itself. Concrete is a brittle material breaking performance was as sudden, its material properties mainly as follows:

(1) Non-elastic volume change, cutting reinforcement, compactness, expansion, and pressure-dependent yield due to the presence of cracks and voids.

(2) Possible non-orthogonal flow property caused by friction.

(3) Brittle strain softening, crack hole may also cause non-linear elasticity, and uninstalling behavior is usually inelastic.

(4) Non-linear creep and its deformation increases over time.

\section{Time-dependent reliability}

Application Example of Time-dependent Reliability Analysis. For compressive strength and elastic modulus, given the incomplete compressive strength and elastic modulus information at various concrete stage (especially the latter), this paper conducts the experimental measurement conversion, makes inverse analysis, engineering analogy and the prediction (gray theory and artificial neural network training) according to the collected data, and solve through mathematical statistics. Specific practice is as follows:

(1) Test measurements

(2) Inverse analysis of statistical experience

(3) Similar projects

(4) Data prediction due to incomplete information at the analysis of time-dependent reliability and prediction by building the gray prediction model or training the artificial neural network through the gray theory.

(5) Statistics and finishing

Due to experimental error and human experience error, there are computed value errors for the above methods, and this paper takes its mean.

Concrete compressive strength and elastic modulus decline over time: due to time-dependent rules of concrete material properties, if concrete strength and elastic modules show obvious time-dependent property, the concrete strength has been improved within 28 days, continue to damage and decay under natural conditions, the concrete elastic modulus measured value reduces nearly $50 \%$ than the stage of just finished construction after 14 years (with measurement error). In the future, the concrete decay gradually becomes flat.

Collect the observational or experimental data of random variables, makes statistical analysis by adopting the mathematical statistics method, solve its distribution rule and distribution characteristic values, adopt the gray theory according to the statistical value at different times, and build GM 
model for time-dependent prediction, in order to provide basis for the calculation of time-dependent reliability.

Obtain the structural resistance through experimental test method and mathematical statistics, build the mechanical model, use the finite element analysis, and calculate the load effect of structure. The structural damage model is also known as the failure criteria.

Build an optimal model for reliability index and solve the optimal reliability solution. Computing model consists of eight-node parameter units. When analyzing the unit, this paper considers the influences of tensile stress and hole on the dam body near the dam heel, the upstream and downstream base rocks respectively take 1 time and 1.5 times of the dam height, the dam unit subdivision is relatively, whereas the bedrock is relatively sparse. The dam is divided into 2660 units and 3588 nodes.

Time-dependent Reliability Analysis of Random Variables. Among the characteristic value of random variables, the variation coefficient is the most sensitive to reliability index, and the mean is relatively less affected. The sensitivity coefficient of random variable is not changed. Where, the sensitivity coefficient means the variation coefficient $\mathrm{c}$, (that is, the ratio of the mean square error and the mean). Observe the impacts of sensitivity coefficient of each random variable on the reliability index.

Clearly, $f_{e}$ variation coefficient has the largest impact on the reliability index, $I_{1}, J_{2}$ variation coefficient has less impact on the reliability index, $\sigma_{1}$ variation coefficient has greater impact on the reliability index, and $f_{e}$ variation coefficient has the largest impact on the reliability index. Through the simplified analysis, deem the variables as $I_{1}, J_{2}$ constants, and only consider the impacts of two random variables $f_{e}$ and $\sigma_{1}$ on reliability index, so as to establish their relationship, and get the mutual relations between variation coefficients of random variables under different reliability indexes. Under the reliability index with engineering requirements, the graphical region enclosed by the variation coefficient curve of random variables is called reliable safety region of random variables. Meanwhile, through the relationship between system input (such as elastic modulus of concrete and density) and output ( $\operatorname{cuch}$ as $\sigma_{1}, I_{1}, J_{2}$ ), this paper may directly establish the relationship between random variables (mechanical properties and external action of materials) and reliability index, in order to solve the traditional method of expressing reliability index by the load effects (such as stress) and resistance. In addition, this paper builds the reliable safety region between various random variables of structural materials.

\section{Conclusions}

Through the above theoretical analysis and practical engineering application, this paper mainly makes the following achievements and results:

1. Compared with the traditional reliability analysis method - semi-empirical and semi-probability, it tries to approach to whole-probability in a more scientific manner, directly deems the structural service as the experience of whole random system, and always applies the probability theory and 
mathematical statistics methods and concepts to analyze the random process in the reliability analysis of entire structural system.

2. This paper systematically discusses the relationship between the system input and output, simulates the training by establishing gray model and artificial neural network, and good results have been achieved for directly transforming the structural performance function from material resistance and load effect to external load random variable..

3. When using sequence development changes of artificial neural network as well as system input and output for fit training, especially for higher-order nonlinear system, this paper can achieve better results.

4. Establish the optimization model for reliability index with the geometric meaning of reliable indexes, apply Muaba software optimization toolbox, and achieve the optimum solution for the structural time-dependent reliability index.

5. Solve the optimal reliability index of optimization model as well as the relationship between input and output of artificial neural network fitting system, and obtain the improved response surface method.

Time-dependent reliability analysis is a systematic multi-disciplinary engineering. Experts, scholars and engineering practitioners concern more about the prediction and evaluation of structural engineering service life. This paper carries out limited related jobs, so the author suggests studying and making efforts from the following aspects:

1. The variability of structural random variable (such as cross-sectional geometry and physical characteristics of materials) parameters have serious impact on the structural stability. Its contribution is generally larger than external excitation (such as load), and thus it is essential to fully consider the random variability of the structural parameters.

2. Considering it is convenient to apply the computer programming to the stochastic finite element analysis, this paper conducts Taylor expansion over the performance function to single-variable term, and the quadratic term is only used for comparison test at the checking point.

3. Describe the random field of stochastic system in a more reasonable way, simulate the near field with finite element, and simulate the far field with combination of boundary element and artificial boundary. Taking into account that the random model can reflect the randomness, discreteness, variability and other uncertainties of structural material geometry, the random field establishment based on local averaging theory, shape function decomposition and perturbation stochastic finite element method based on related structural fitting have in-depth research value.

\section{Acknowledgment}

This work was supported by Project on professional and characteristical construction of Jiangxi province 2010 (Civil Engineering) and Project on the planning and construction of disciplines in Jiangxi University of Technology (Structure Engineering)

\section{References}

[1] Richards Jr R, Mark R. Gelatin models for photoelastic analysis of gravity structures[J]. Experimental Mechanics, 1966, 6(1): 30-38. 
[2] Ardito R, Cocchetti G. Statistical approach to damage diagnosis of concrete dams by radar monitoring: Formulation and a pseudo-experimental test[J]. Engineering structures, 2006, 28(14): 2036-2045.

[3] Shutian L, Grandhi R V. A fast Fourier transformation based second order reliability method[J]. ACTA MECHANICA SOLIDA SINICA-CHINESE EDITION-, 2001, 22(4): 387-393.

[4] Wei H, Yang H S, Wu L. Gravity dam safety reliability analysis based on artificial neural network[J]. Journal of Hohai University: Natural Sciences, 2011, 39(4): 415-420.

[5] Fattahi H, Shojaee S, Farsangi M A E, et al. Hybrid Monte Carlo simulation and ANFIS-subtractive clustering method for reliability analysis of the excavation damaged zone in underground spaces[J]. Computers and geotechnics, 2013, 54: 210-221.

[6] Chen H. Seismic safety of high concrete dams[J]. Earthquake Engineering and Engineering Vibration, 2014, 13(1): 1-16.

[7] Lahmer T. Optimal experimental design for nonlinear ill-posed problems applied to gravity dams[J]. Inverse Problems, 2011, 27(12): 125005.

[8] Grossmann A, Morlet J. Decomposition of Hardy functions into square integrable wavelets of constant shape[J]. SIAM journal on mathematical analysis, 1984, 15(4): 723-736.

[9] Johansson R, Kronberg E. Stability analysis of the hydropower arch dam Jinping I[J]. 2011.

[10] Wang Y, Li X, Wu Y F, et al. Experimental study on meso-damage cracking characteristics of RSA by CT test[J]. Environmental Earth Sciences, 2014: 1-14. 